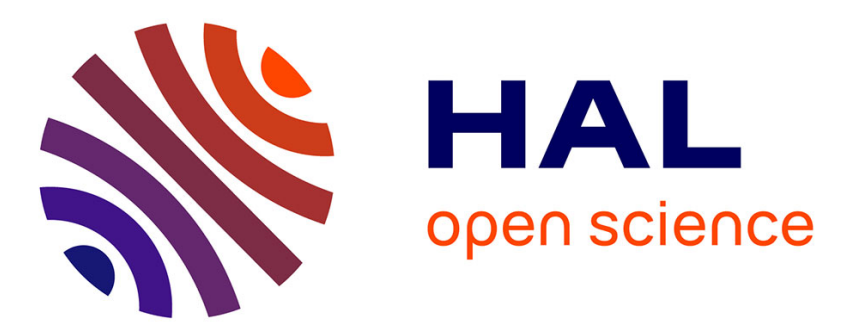

\title{
Experimental investigation and analytical description of a vibro-impact NES coupled to a single-degree-of-freedom linear oscillator harmonically forced
}

\author{
Giuseppe Pennisi, Cyrille Stephan, Etienne Gourc, Guilhem Michon
}

\section{To cite this version:}

Giuseppe Pennisi, Cyrille Stephan, Etienne Gourc, Guilhem Michon. Experimental investigation and analytical description of a vibro-impact NES coupled to a single-degree-of-freedom linear oscillator harmonically forced. Nonlinear Dynamics, 2017, 88 (3), pp.1769 - 1784. 10.1007/s11071-017-3344-1 . hal-01829677

\section{HAL Id: hal-01829677 \\ https://hal.science/hal-01829677}

Submitted on 4 Jul 2018

HAL is a multi-disciplinary open access archive for the deposit and dissemination of scientific research documents, whether they are published or not. The documents may come from teaching and research institutions in France or abroad, or from public or private research centers.
L'archive ouverte pluridisciplinaire HAL, est destinée au dépôt et à la diffusion de documents scientifiques de niveau recherche, publiés ou non, émanant des établissements d'enseignement et de recherche français ou étrangers, des laboratoires publics ou privés. 


\title{
Experimental Investigation and Analytical Description of a Vibro-Impact NES Coupled to a Single-Degree-of-Freedom Linear Oscillator Harmonically Forced
}

\author{
Giuseppe Pennisi*1 ${ }^{*}$ Cyrille Stephan ${ }^{\dagger 2}$, Etienne Gourc ${ }^{\ddagger 3}$, and \\ Guilhem Michon ${ }^{\S}$ \\ ${ }^{1}$ ONERA The French Aerospace Lab, Université de Toulouse, ICA, ISAE \\ ${ }^{2}$ ONERA The French Aerospace Lab \\ ${ }^{3}$ Université de Toulouse, ICA, INSA \\ ${ }^{4}$ Université de Toulouse, ICA, ISAE
}

Nonlinear Dynamics (2017), doi: 10.1007/s11071-017-3344-1.

The final publication is available at

http://link.springer.com/article/10.1007/s11071-017-3344-1

\begin{abstract}
In this paper the dynamics of a system composed of a harmonically forced single-degree-of-freedom linear oscillator coupled to a Vibro-Impact Nonlinear Energy Sink (VI-NES) is experimentally investigated. The mass ratio between the VI-NES and the primary system is about $1 \%$. Depending on the external force's amplitude and frequency, either a Strongly Modulated Response (SMR) or a constant amplitude response (CAR) is observed. In both cases an irreversible transfer of energy occurs from the linear oscillator towards the VI-NES: process known in literature as passive Targeted Energy Transfer (TET). Furthermore, the problem is analytically studied by using the method of multiple scales. The obtaineSlow Invariant Manifold (SIM) shows the existence of a stable and of an unstable branch of solutions, as well as of an energy threshold (a saddle-node bifurcation) for the solutions to appear. Subsequently the fixed points of the problem are calculated. When a stable fixed point is reached, the system is naturally drawn to it and a CAR is established, whereas when no stable point is attained, the system exhibits a SMR regime. Finally a good correlation between the experimental and the analytical results is presented.
\end{abstract}

\footnotetext{
*giuseppe.pennisi@onera.fr

$\dagger_{\text {cyrille.stephan@onera.fr }}$

¥gourc@insa-toulouse.fr

§guilhem.michon@isae.fr
} 


\section{Introduction}

A Nonlinear Energy Sink (NES) is defined as a vibration absorber with a relatively light mass, nonlinearly attached to a primary system whose vibrations are to be mitigated. The use of a NES as a vibration absorber has been object of interest in the field of nonlinear dynamics in the last decade. Studies have shown that, if compared to the classical linear Tuned Mass Damper (TMD), it could be efficient in a broader frequency range and for a smaller addition of mass to the primary system [1-3]. It has been shown that the nonlinear attachments can lead to an irreversible energy transfer from the primary system towards the NES, this process is known as Targeted Energy Transfer (TET) or energy pumping $[4,5]$.

Experimental works have investigated this phenomenon and are presented in [6-8]. They have shown that the dynamics which governs the energy transfer phenomenon is a 1:1 resonance capture between the primary system and the NES. An important feature of this kind of devices is that they do not have a preferrable frequency of oscillation because of their intrinsic nonlinear nature. Thanks to this characteristic, they are able to tune themselves to the primary system's frequency. TET under external forcing has been investigated theoretically [9] and experimentally [10] showing that in addition to the steadystate-constant-amplitude-response regime, another type of response can arise which is referred to as Strongly Modulated Response (SMR). NESs have also been studied when applied to passive control of instabilities. In [11] a NES was used to control the limit cycles of a Van der Pol oscillator. In [12-15] it was used to suppress aeroelastic instabilities.

Most of the works mentioned so far have dealt with a nonlinearity represented by a cubic stiffness. The principle consists in using the geometric nonlinearity of an elastic element to attain a restoring force proportional to the cube of the displacement. Nevertheless, the nature of the nonlinearity may theoretically be of any kind. Later studies have explored other ideas such as: non-polynomial functions [16], multiple states of equilibrium [17], non-smooth functions and Vibro-Impacts [18-21]. Many of the first works on Vibro-Impacts were based on numerical simulations. Recently, similarly to what was used for smooth nonlinearities, an analytical approach adopting the multiple-scale analysis has been proposed in [22] for impulsive forces, and extended to the case of a harmonic forcing in [23]. In [24] preliminary experimental observations of the different regimes of response has been achieved for a VI-NES applied to a harmonically forced linear oscillator.

In this paper the experimental and the analytical study of the response of a system composed of a Vibro-Impact NES coupled to a single- degree-of-freedom linear oscillator is carried out. The main purpose of this work is to bridge the theoretical and experimental studies, in order to reach a comparison between the analytical results and the experimental observations. The study carried out in [24] is used as an important starting point to broaden the general understanding of the VI-NES. In [24], experimental investigations have shown how such a system may exhibit different types of response as the external forcing changes 
in amplitude and/or frequency. It also has been shown how an analytical study based on the multiple-scale analysis is able to describe this kind of behavior.

Thanks to these initial observations, it can be stated that the forcing term plays a crucial role in the problem. In this paper the analytical treatment is slighlty different than in [24], in order to take into account the forcing term since the very first steps of the multiple-scale expansion. As for the experimental part, in order to prove the efficacy of the absorber for different frequencies, the same VI-NES as in [24] is used, but applied to a different primary system having a higher natural frequency. Moreover, the use of a bearing slider is avoided as it turned out being a source of perturbation for the experiments. Four levels of force are tested and for each level a frequency sweep is performed; this leads to a study of the response of the VI-NES over a considerable range of forcing cases.

Finally, the analytical model is used to explain the behavior experimentally observed and a comparison between the experimental and the analytical results is presented. In addition, the influence of the design parameters of the VI-NES on the system's response is discussed thanks to some analytical examples.

\section{Experimental tests}

The experimental study has been conducted aiming to observe the behavior of the system and to explore the existing different types of response which can arise and how they are related to the external forcing in terms of magnitude and frequency. This phase of experimental investigation will be subsequently exploited and the observations explained by the analytical study of the system.

The experimental apparatus is shown in Fig.1. It is constituted of a singledegree-of-freedom linear oscillator (LO) to which the VI-NES is attached. The LO is harmonically forced by an electrodynamic shaker.
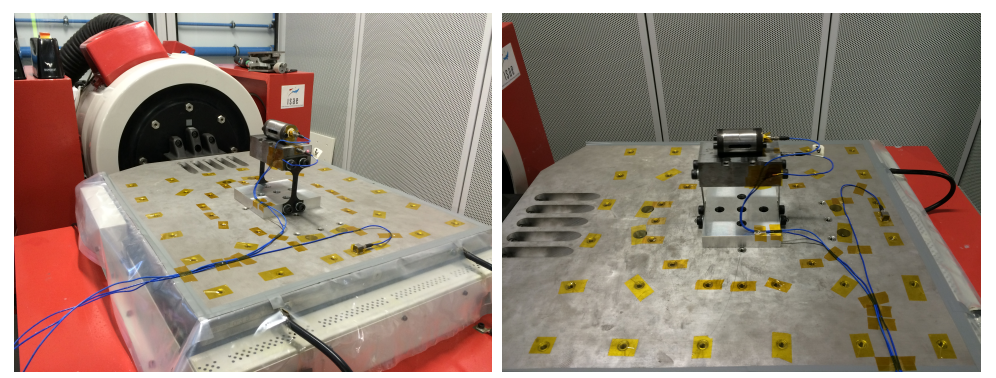

Figure 1: The LO coupled to the VI-NES: the prototype installed on the vibrating table.

Figure 2 displays the tested prototype and its schematic: the primary mass $M$ is connected to its base by means of two flexible blades. The base is then screwed to the vibrating table. On the primary mass, a cylinder is placed and 

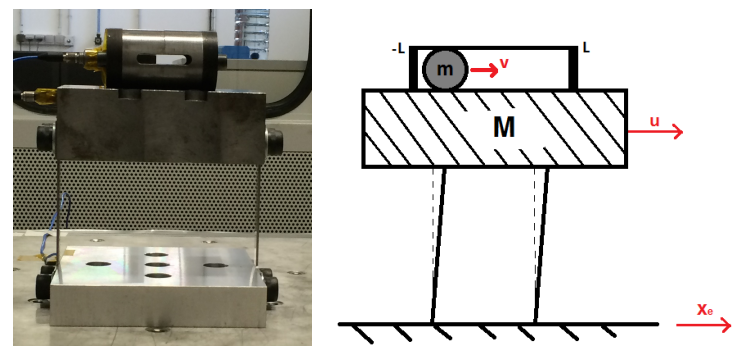

Figure 2: The prototype (left) and its schematic (right).

the small VI-NES mass is free to roll inside. The cylinder is pierced on the sides in order to make the air effect negligible and to visually observe the motion of the ball. Firstly a dynamic identification of the primary system is performed. The system is excited by a swept-sine external force with constant amplitude and the displacement of the primary mass is measured by means of a Laser Doppler Vibrometer (LVD). The use of a LVD rather than an accelerometer has been dictated by the fact that the impacts would drastically perturb the signals delivered by an accelerometer.

\begin{tabular}{|c|c|c|c|}
\hline$f_{0}[\mathrm{~Hz}]$ & $K[N / m]$ & $\lambda[N s / m]$ & $\xi$ \\
\hline \hline 21.18 & 67421 & 8.566 & 0.008 \\
\hline
\end{tabular}

Table 1: Modal parameters of primary system.

The modal parameters of the primary system are shown in Tab.1 and the mass values and their ratio in Tab.2. Several tests were performed on the primary structure at different levels of base acceleration between $0.1-0.5 \mathrm{~g}$. They showed that the primary system was not perfectly linear and that a slight variation in the modal parameters occurred as the external force varied. However this was a weakly nonlinear behavior that did not affect the purpose of the tests. It is important to highlight the particularly small mass ratio between the VI-NES and the primary system: less than $1 \%$. Once the primary system has been identified, we arm the VI-NES by inserting the small mass into the cavity and we carry out some swept-sine tests for four different levels of forcing: $[0.2 ; 0.3 ; 0.4 ; 0.5] \mathrm{g}$; the displacement is measured by the LVD and the spectra are then obtained. The fact of using a large-mass shaker permits the shaker not

\begin{tabular}{|c|c|c|}
\hline$M[k g]$ & $m[k g]$ & $\epsilon=m / M$ \\
\hline \hline 3.807 & 0.032 & $0.84 \%$ \\
\hline
\end{tabular}

Table 2: Mass values and their ratio $\epsilon$. 


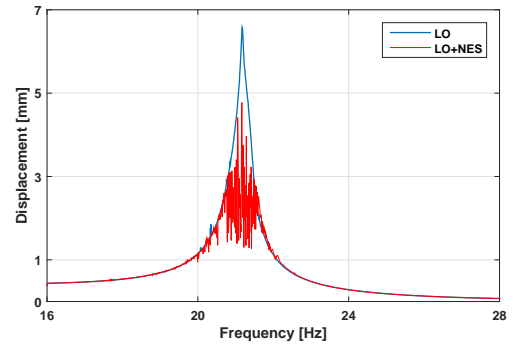

(a) $\ddot{x}_{e}=0.2 g$

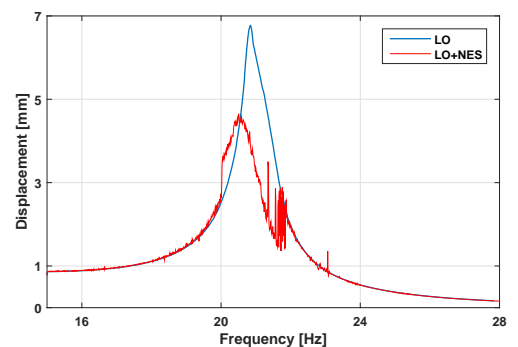

(c) $\ddot{x}_{e}=0.4 g$

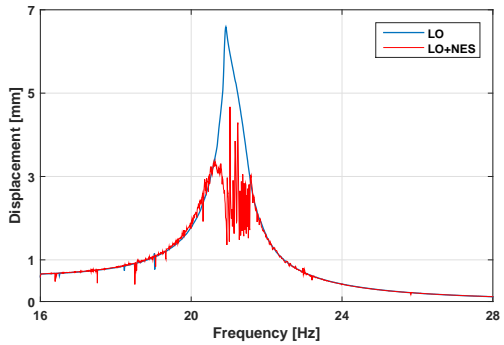

(b) $\ddot{x}_{e}=0.3 g$

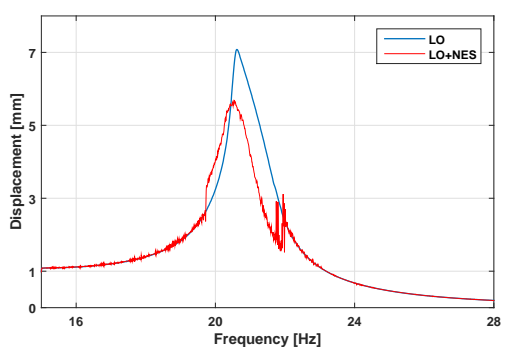

(d) $\ddot{x}_{e}=0.5 g$

Figure 3: Experimental spectra of the primary mass displacement with (red) and without (blue) the VI-NES for four different levels of external forcing. It can be observed that the VI-NES is inactive for values of the primary mass' displacement under a certain threshold $(\approx 2.8 \mathrm{~mm})$. When the threshold is reached, the VI-NES turns active and two different regimes may occur: either a constant amplitude response (CAR) or a strongly modulated response (SMR).

to be perturbed when it goes through the natural frequency of the LO during the swept-sine.

Fig.3 shows the displacement spectra for the system with and without the VI-NES for four levels of external excitation: $[0.2 ; 0.3 ; 0.4 ; 0.5]$ g. Fig.4 shows the measured displacement for two different frequencies at the same amplitude of external acceleration.

Firstly, we can observe that a threshold in the amplitude of the primary mass' oscillations exists for the VI-NES to become active: we will refer to it as activation threshold. Secondly, the system can exhibit two types of response and, depending on the magnitude and the frequency of the external forcing, either one or another may appear.

The responses can qualitatively be classified as:

- - Idle VI-NES: no impacts occur and the primary system's dynamics is not perturbed by the presence of the VI-NES: the amplitude of the LO's oscillations is lower than the activation threshold.

- - Constant Amplitude Response (CAR): the VI-NES is stably active and 
the amplitude of the primary mass displacement remains constant (fig.4 left).

- - Strongly Modulated Response (SMR): the primary system goes through alternatively increasing and decreasing amplitude cycles and as a consequence the fast oscillations appear to be modulated. This behavior is caused by a cyclical activation/deactivation of the VI-NES (fig.4 right).
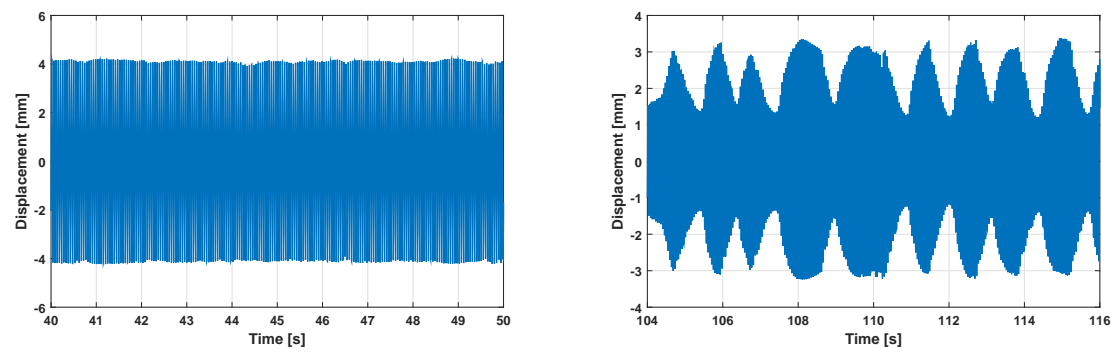

Figure 4: Time series: constant amplitude response $-\ddot{x}_{e}=0.4 g-f=20.9 \mathrm{~Hz}$ (left) and strongly modulated response $-\ddot{x}_{e}=0.4 g-f=21.5 \mathrm{~Hz}$ (right).

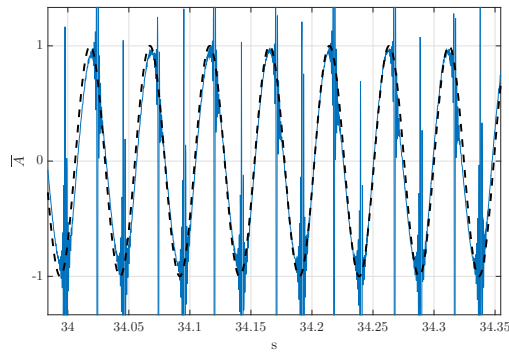

Figure 5: Experimental impacts detection: acceleration of the LO measured by an accelerometer placed on the VI-NES in order to capture the impact instants. The peaks represent the shocks occurring throughout the oscillations of the LO, highlighted by the dashed line. $\bar{A}$ denotes the normalized acceleration of the LO. It can be seen as two impacts per cycle occur.

The VI-NES seems to well accomplish its task as a vibration absorber since the amplitude of the response is reduced nearby the resonance of the primary system. This is the proof that a Targeted Energy Transfer occurs from the LO towards the VI-NES and that the energy is then dissipated by the impacts. It is important to highlight that this goal has been achieved although a proper sizing process of the VI-NES has not been carried out and with a significantly small mass ratio $\epsilon=0.84 \%$. This result proves that the VI-NES is able to 


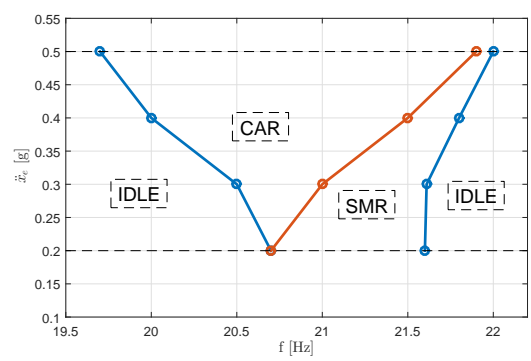

Figure 6: Map showing the regime zones of the responses experimental observed as functions of the external excitation's magnitude and frequency.

automatically tune itself to the primary system. This is a relevant general feature of nonlinear absorbers due to their lack of a natural frequency. However, whereas for a cubic stiffness NES the absence of a natural frequency is a delicate goal to reach [10], for a VI-NES it appears to be as an evident outcome. Still, we remind that the primary objective of this experimental study was not to seek the optimal performance but rather to investigate the qualitative behavior of the VI-NES.

Looking at the spectra of Fig.3 one can draw the conclusion that there exists a criterion on primary mass displacement in order to activate the VINES. Indeed a threshold in amplitude/energy is observed beyond which the VI-NES goes through a 1:1 resonance with respect to the LO. The terminology 1:1 resonance is used to maintain a correlation with the cubic stiffness NES, even though in this context it means that two impacts per oscillation cycle of the LO occur. This can be seen in Fig. 5 where the signal of an accelerometer placed on the primary mass is shown. The peaks represent the shocks occurring two times per period throughout the oscillations of the LO.

Fig. 6 shows a map illustrating the nature of the observed responses as a function of the magnitude and the frequency of the external forcing. For a low level of external forcing only a SMR is registered through the whole range of frequencies where the VI-NES is active. As the external force increases, a CAR appears at the lowest frequencies of the activation range and the SMR tends to disappear. When high levels of external forcing are reached the CAR becomes the prevalent, if not the only, type of response detected. It is therefore clear that for a given VI-NES the kind of response which might arise is a function of the magnitude and the frequency of the external forcing. In the next section this behavior will be explained by an analytical analysis of the mathematical problem. 


\section{Analytic treatment}

In this section the mathematical problem associated to the VI-NES is studied. An analytical treatment is presented, in which the multiple-scale method is used in order to obtain the Slow Invariant Manifold (SIM) and the fixed points of the problem. The multiple-scale is a perturbation method used to construct uniformly valid approximations to the solution of the problem under consideration. This is done by introducing fast-scale and slow-scale variables and treating these variables as independent. This method is particularly useful when the problem is characterized by having multiple dynamics, each with their own scales. In our problem one scale is associated to the fast oscillations and another to their amplitude's slow modulation.

The approach followed is mainly based on the work of Gendelman [22], where the analytic treatment of an unforced system with a VI-NES is presented. Although not original, these derivations are required for the sake of completeness of the exposition. Here we extend the treatment to a damped and forced system such as in [24], with the difference that the forcing term is not hypothetically supposed to be of order 1 but considered since order 0 in the multiple-scale expansion.

The schematic of the model is presented in Fig.2. We define the variables $u, v$, and $x_{e}$ as the displacements of the primary mass $M$, of the NES mass $m$ and of the base respectively. Before going through the study of the equations of motion, we model the impacts by using the Newton's experimental law and the momentum conservation principle. This model, although quite simple, is able to represent the physics of the impacts by means of only one parameter: the restitution coefficient $r$.

$$
\begin{aligned}
\dot{u}\left(t_{j}^{+}\right)-\dot{v}\left(t_{j}^{+}\right) & =-r\left(\dot{u}\left(t_{j}^{-}\right)-\dot{v}\left(t_{j}^{-}\right)\right) \\
M \dot{u}\left(t_{j}^{+}\right)+m \dot{v}\left(t_{j}^{+}\right) & =M \dot{u}\left(t_{j}^{-}\right)+m \dot{v}\left(t_{j}^{-}\right)
\end{aligned}
$$

Where $t_{j}^{+}$and $t_{j}^{-}$are the time instants after and before the $j^{t h}$ impact respectively. Eq.(1) provides a relation for the relative velocities of the two colliding masses after and before the impact by use of the restitution coefficient $0<r<1$. Then, in the limit cases, impacts can be characterized as perfectly elastic $r=1$ or perfectly inelastic $r=0$. Eq.(2) expresses the momentum conservation throughout an impact. Each mass experiences an instantaneous momentum variation that can be represented by a Heaviside step function. The derivative of momentum with respect to time is the force acting through the impact. Thus in (3) we express the force each mass is subjected to as a Dirac delta function, which represents the distributional derivative of the Heaviside step function. In Eq.(3) the summation includes all the impacts that occur over the time. Since all the terms of the summation are Dirac delta functions, it means that they are always zero except when an impact occurs. When this happens, only the one term of the sum associated to that specific impact takes a value different than zero, as its delta becomes one. 
Finally the equations of motion can be written as:

$$
\begin{gathered}
M \ddot{u}+\lambda \dot{u}+K u+\frac{M m(1+r)}{M+m} \sum_{j} \dot{w}^{-} \delta_{j}^{-}=K x_{e}+\lambda \dot{x}_{e} \\
m \ddot{v}-\frac{M m(1+r)}{M+m} \sum_{j} \dot{w}^{-} \delta_{j}^{-}=0
\end{gathered}
$$

Where $M, \lambda$ and $K$ are the mass, damping and stiffness of the primary system, $m$ is the mass of the VI-NES and $r$ the restitution coefficient.

Or:

$$
\begin{gathered}
\ddot{u}+2 \omega_{0} \xi \dot{u}+\omega_{0}^{2} u+\frac{m(1+r)}{M+m} \sum_{j} \dot{w}^{-} \delta_{j}^{-}=\omega_{0}^{2} x_{e}+2 \omega \xi \dot{x}_{e} \\
\epsilon \ddot{v}-\frac{m(1+r)}{M+m} \sum_{j} \dot{w}^{-} \delta_{j}^{-}=0
\end{gathered}
$$

Where $\omega_{0}^{2}=K / M, 2 \omega_{0} \xi=\lambda / M$ and $\epsilon=m / M$.

Defining the barycentric coordinates $X$ and $w^{1}$

$$
\begin{aligned}
X & :=u+\epsilon v \\
w & :=u-v
\end{aligned}
$$

And the following new variables:

$$
\tau:=t \frac{\omega_{0}}{\sqrt{1+\epsilon}} \quad \gamma:=\frac{2 \xi}{\sqrt{1+\epsilon}}
$$

We end up with a system of two equations for $X$ and $w$, where the subscript $\tau$ denotes the partial derivative $\frac{\partial}{\partial \tau}$. They are up to now exact equations since no approximation has been made.

$$
\begin{gathered}
X_{\tau \tau}+\gamma X_{\tau}+\epsilon \gamma w_{\tau}+X+\epsilon w=(1+\epsilon) x_{e}+\gamma(1+\epsilon) x_{e_{\tau}} \\
w_{\tau \tau}+\epsilon \gamma w_{\tau}+\gamma X_{\tau}+\epsilon w+X+(1+r) \sum_{j} w_{\tau}^{-} \delta_{j}^{-}=(1+\epsilon) x_{e}+(1+\epsilon) \gamma x_{e_{\tau}}
\end{gathered}
$$

\subsection{Multiple-scale analysis}

Assuming that the mass of the VI-NES $m$ is small with respect to the primary mass $M, \epsilon=m / M<<1$ can be used as a small parameter in the multiple scales analysis [25].

$$
\begin{gathered}
\tau_{k}=\epsilon^{k} \tau, k=0,1, \ldots ; \quad \frac{d}{d t}=\frac{\partial}{\partial \tau_{0}}+\epsilon \frac{\partial}{\partial \tau_{1}}+\ldots ; \\
X=X_{0}\left(\tau_{0}, \tau_{1}, \ldots\right)+\epsilon X_{1}\left(\tau_{0}, \tau_{1}, \ldots\right)+\ldots ; \\
w=w_{0}\left(\tau_{0}, \tau_{1}, \ldots\right)+\epsilon w_{1}\left(\tau_{0}, \tau_{1}, \ldots\right)+\ldots ;
\end{gathered}
$$

\footnotetext{
${ }^{1}$ The symbol $:=$ meaning equals by definition
} 
Bearing in mind that $\epsilon<<1$, the parameter $\gamma$ can be expressed as a firstorder Taylor polynomial:

$$
\begin{aligned}
\gamma=\frac{2 \xi}{\sqrt{1+\epsilon}} & =2 \xi(1+\epsilon)^{-1 / 2} \\
& \simeq 2 \xi\left(1-\frac{\epsilon}{2}\right)
\end{aligned}
$$

The same is done with the external forcing term:

$$
\begin{aligned}
x_{e} & =\bar{X} \sin (\omega t) \\
& =\bar{X} \sin \left(\frac{\omega}{\omega_{0}}(1+\epsilon)^{1 / 2} \tau\right) \\
x_{e} & \simeq \bar{X} \sin \left(\frac{\omega}{\omega_{0}} \tau_{0}\right)+\frac{1}{2} \frac{\omega}{\omega_{0}} \tau_{1} \bar{X} \cos \left(\frac{\omega}{\omega_{0}} \tau_{1}\right) \epsilon
\end{aligned}
$$

Substituting (8), (9) and (10) into (7) and only keeping the zero-order terms:

$$
\begin{gathered}
\frac{\partial^{2} X_{0}}{\partial \tau_{0}^{2}}+2 \xi \frac{\partial X_{0}}{\partial \tau_{0}}+X_{0}=X_{e_{0}}+2 \xi \frac{\partial X_{e_{0}}}{\partial \tau_{0}} \\
\frac{\partial^{2} w_{0}}{\partial \tau_{0}^{2}}+2 \xi \frac{\partial X_{0}}{\partial \tau_{0}}+X_{0}+(1+r) \sum_{j} \frac{\partial w_{0}^{-}}{\partial \tau_{0}} \delta_{j}^{-}=X_{e_{0}}+2 \xi \frac{\partial X_{e_{0}}}{\partial \tau_{0}}
\end{gathered}
$$

Where $X_{e_{0}}=\bar{X} \sin \left(\Omega \tau_{0}\right)$ with $\Omega=\frac{\omega}{\omega_{0}}$.

By considering the experimental values we identified $(\xi \approx \epsilon \approx 0.8 \%$ ) we can reasonably assume that $\xi=\frac{\lambda}{2 \omega_{0} M}$ has the same order as $\epsilon$ and therefore that $\xi=O(\epsilon)$. Consequently, the terms multiplied by $\xi$ in Eq.(11) can be neglected. That yields:

$$
\begin{gathered}
\frac{\partial^{2} X_{0}}{\partial \tau_{0}^{2}}+X_{0}=X_{e_{0}} \\
\frac{\partial^{2} w_{0}}{\partial \tau_{0}^{2}}+X_{0}+(1+r) \sum_{j} \frac{\partial w_{0}^{-}}{\partial \tau_{0}} \delta_{j}^{-}=X_{e_{0}}
\end{gathered}
$$

Alternatively, one can say that solutions of Eq.(12) will be very close to solutions of Eq.(11) given the small influence of $\xi$. Then the two equations of Sys.(12) can be solved for $X_{0}$ and $w_{0}$. Here we follow the ansatz given by Gendelman in [22] and [23] with the exception that the forcing term is present at the $\epsilon^{0}$ order. That leads to the following expression for $X_{0}$ :

$$
X_{0}=C\left(\bar{X}, \Omega, \tau_{1}\right) \sin \left(\Omega \tau_{0}+\psi\left(\tau_{1}\right)\right)
$$

$C$ and $\psi$ are the amplitude and the phase of $X_{0}$. The amplitude $C$ is a function of the external forcing $X_{e_{0}}$ and terms of order-1. The phase $\psi$ is a function of order-1 terms as it depends on damping $\xi$.

Substituting (13) in the second equation of (12) we obtain:

$$
\frac{\partial^{2} w_{0}}{\partial \tau_{0}^{2}}+(1+r) \sum_{j} \frac{\partial w_{0}^{-}}{\partial \tau_{0}} \delta_{j}^{-}=A \sin \left(\Omega \tau_{0}+\theta\right)
$$


Where $A \sin \left(\Omega \tau_{0}+\theta\right)$ is the sum of $X_{0}$ and $X_{e 0}$. These two terms are two sinus with the same frequency $\Omega$ and shifted in phase by $\psi$. It can be shown that the sum of them is a single sinus with a new amplitude $A$ and phase $\theta$ to be determined.

A solution for $w_{0}$ in the following form is sought:

$$
w_{0}=-\frac{A(C, \bar{X}, \psi)}{\Omega^{2}} \sin \left(\Omega \tau_{0}+\theta\right)+f\left(\tau_{0}, \tau_{1}\right)
$$

Inserting (15) into (14):

$$
\frac{\partial^{2} f}{\partial \tau_{0}^{2}}+(1+k) \sum_{j}\left[\left.\frac{\partial f}{\partial \tau_{0}}\right|_{\tau_{0}^{-}}-\frac{A}{\Omega} \cos \left(\Omega \tau_{0}+\theta\right)\right] \delta_{j}^{-}=0
$$

Assuming that TET occurs in an established state of 1:1 resonance between the VI-NES and the LO and neglecting the transient process leading to 1:1 resonance, the particle will move symmetrically and at the same frequency of the LO. A form of $f$ representing the "free flights" interrupted by the impacts is:

$$
f\left(\tau_{0}, \tau_{1}\right)=\frac{2 \alpha}{\pi} \arcsin \left(\cos \left(\Omega\left(\tau_{0}-\eta\right)\right)\right)
$$

Where $t_{j}=\eta+\pi j, j=1,2, \ldots$ are the unknown impact instants. A schematic representation of $f$ is presented in Fig.7.

Substituting (17) into (16) and integrating over a small interval around time $\tau_{0}=\eta$ :

$$
-\frac{4 \alpha \Omega}{\pi}+(1+r)\left(\frac{2 \alpha \Omega}{\pi}-\frac{A}{\Omega} \cos (\Omega \eta+\theta)\right)=0
$$

And finally:

$$
-A \cos (\Omega \eta+\theta)=\Omega^{2} \frac{2(1-r)}{\pi(1+r)} \alpha=\Omega^{2} \sigma \alpha
$$

Where $\sigma=\frac{2(1-r)}{\pi(1+r)}$. One more relation between $A$ and $\alpha$ can be found by considering the expression (15) evaluated when an impact occurs: $\tau_{0}=\eta$ and $w= \pm L$.

$$
-\frac{A}{\Omega^{2}} \sin (\Omega \eta+\theta)+\alpha=L
$$

Finally we have:

$$
\begin{aligned}
& A_{\Omega} \cos (\Omega \eta+\theta)=\sigma \alpha \\
& A_{\Omega} \sin (\Omega \eta+\theta)=L-\alpha
\end{aligned}
$$

With $A_{\Omega}=-A / \Omega^{2}$.

The two equations (21) allows one to analytically define the Slow Invariant Manifold of the problem:

$$
\alpha=\frac{L \pm \sqrt{L^{2}-\left(1+\sigma^{2}\right)\left(L^{2}-A_{\Omega}^{2}\right)}}{1+\sigma^{2}}
$$


Or:

$$
\alpha=\frac{L \pm \sqrt{1+\sigma^{2}} \sqrt{A_{\Omega}^{2}-A_{\Omega_{m i n}}^{2}}}{1+\sigma^{2}}
$$

Where $A_{\Omega_{\text {min }}}=\frac{\sigma L}{\sqrt{1+\sigma^{2}}}$ is a minimum value of amplitude $A / \Omega^{2}$ for a $1: 1$ resonance to be established.

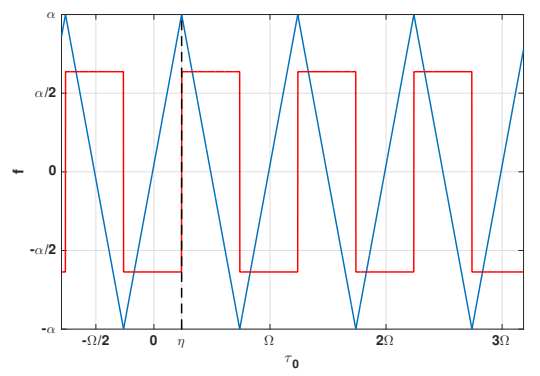

Figure 7: Sketch of function $f$ according to eq.(17) (blue) and its derivative (red).

Eq.(22) defines the Slow Invariant Manifold of the system and a graphical representation for the couple of parameters $r=0.65$ and $L=15 \mathrm{~mm}$ is displayed in Fig.8. A first important piece of information we can draw from Eq.(22) is that a minimum for $A_{\Omega}$ exists for solutions to appear. In order to have a physical meaning of the two variables of the SIM, $A_{\Omega}$ and $\alpha$ can be thought as respectively the LO's and the NES' oscillation amplitudes. Then the lower threshold is actually a minimum amount of LO's energy (oscillations amplitude) the VI-NES needs to turn active. As previously mentioned this is a typical feature of nonlinear systems $[2,3,5]$. Moreover, information about stability of solutions can be obtained from $\epsilon^{0}$-equations. Indeed a stability analysis can be performed by means of the Poincare Map which reveals that the SIM is composed by two branches of solutions: one stable and one unstable. We will not describe the analysis here but only suggest that the reader refer to [26] for a detailed mathematical description. Indeed, to have the same formal problem as in [26] we just need to rearrange the second equation of Sys.(12) as:

$$
\begin{array}{ll}
\frac{\partial^{2} w_{0}}{\partial \tau_{0}^{2}}=A \sin \left(\Omega \tau_{0}+\theta\right) & \left|w_{0}\right|<L \\
\frac{\partial w_{0}^{+}}{\partial \tau_{0}}=-r \frac{\partial w_{0}^{-}}{\partial \tau_{0}} & \left|w_{0}\right|=L
\end{array}
$$

As it will be explained more in detail in the next section, from a mathematical point of view this threshold represents a saddle-node bifurcation beyond which two branches of solutions appear, one stable and one unstable.

The SIM strongly depends on the design parameters length $L$ and restitution coefficient $r$ as graphs in Fig.9 show. Particularly, it is interesting to highlight 
how in the case of $r=1$ the value for $A_{\Omega_{\min }}$ is zero and the corresponding value of $\alpha$ is equal to half the tube length, i.e. the distance gap between the ball and the tube before impacting. This is physically explicable as $r=1$ means no loss of energy during the impacts, the state with two impacts per cycle could therefore be maintained without the primary mass moving, i.e. with no input of energy into the system.

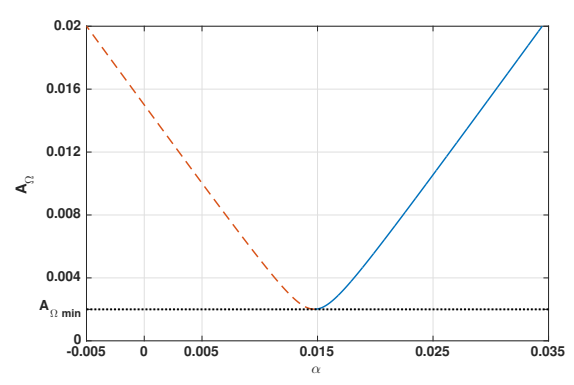

Figure 8: Slow Invariant Manifold $-r=0.65, L=15 \mathrm{~mm}$. Two branches of solutions exist: one stable (solid blue) and one unstable (dashed red). $A_{\Omega_{\min }}$ is the minimum value of $A_{\Omega}$ for the solutions to appear; this point mathematically represents a saddle-node bifurcation point.
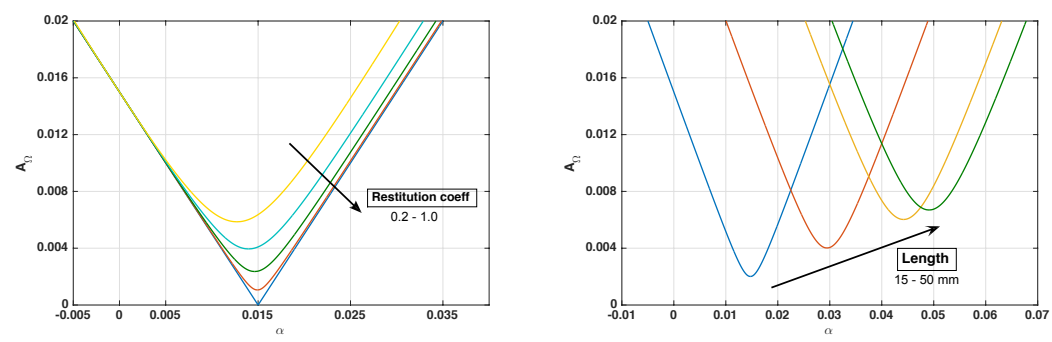

Figure 9: Evolution of $\epsilon^{0}$-SIM for constant $L=15 \mathrm{~mm}$ as the restitution coefficient varies (left) and for constant $r=0.65$ as the length varies (right).

Once the SIM obtained, we push further our analysis and study Sys. (7) at the $\epsilon^{1}$ scale in order to study the evolution of the system on the SIM as the amplitude and the frequency of the forcing term vary.

Only keeping the $\epsilon^{1}$-order terms, the first equation of Sys.(7) becomes:

$$
\frac{\partial^{2} X_{1}}{\partial \tau_{0}^{2}}+X_{1}=-2 \frac{\partial^{2} X_{0}}{\partial \tau_{0} \partial \tau_{1}}-\gamma \frac{\partial X_{0}}{\partial \tau_{0}}-\frac{\partial^{2} X_{0}}{\partial \tau_{0}^{2}}-w_{0}+Y \cos \left(\Omega \tau_{0}+\beta\left(\tau_{1}\right)\right)
$$


Where $Y \cos \left(\Omega \tau_{0}+\beta\left(\tau_{1}\right)\right)=\bar{X} \sin \left(\Omega \tau_{0}\right)+\gamma \Omega \bar{X} \cos \left(\Omega \tau_{0}\right)$.

After substituting (13) into (24) and eliminating secular terms we are able to find the approximate solution provided by the multiple-scale analysis. By equaling derivatives with respect to time to zero, the fixed points are obtained. These steps are not reported here for the sake of conciseness and can be found in [24]. Finally we obtain a fourth order polynomial relating $A$ and $\alpha$ :

$$
a_{2} A^{4}+a_{1} A^{2}+a_{0}=0
$$

Where the coefficients $a_{0}, a_{1}, a_{2}$ are functions of $\alpha$ :

$$
\begin{aligned}
& a_{0}=\frac{64}{B^{2} \pi^{4}}\left(\sigma^{2}+1\right) \alpha^{4}-\frac{128}{B^{2} \pi^{4}} \alpha^{3}+\frac{64}{B^{2} \pi^{4}} \alpha^{2} \\
& a_{1}=\frac{32}{B^{2} \pi^{2}}\left(\frac{\mu \sigma}{2}+\delta\right) \alpha^{2}-\frac{32 \delta}{B^{2} \pi^{2}} \alpha-1 \\
& a_{2}=\frac{\left(\mu^{2}+4 \delta^{2}\right)}{B^{2}}
\end{aligned}
$$

Where $\delta$ is the detuning parameter defined as $\Omega=1+\epsilon \delta, B$ is the scaled external amplitude term $B=\frac{\bar{X}}{L \epsilon}$ and $\mu$ is the scaled damping coefficient $\mu=2 \xi / \epsilon$. It can be seen that, unlike the SIM, the fixed points expression depends on the external excitation's amplitude $\bar{X}$ and frequency $\Omega$ through the variables $B$ and $\delta$. It is also important to notice that, as it was for the SIM, the fixed points expression is a function of the design parameters tube's length $L$ and restitution coefficient $r$ (through $\sigma$ ).

The solutions of the problem will satisfy both Eq.(22) and Eq.(25) and can graphically be identified by the intersections of these two curves on the plane $A_{\Omega}-\alpha$. Alternatively, one can say that the fixed points of Eq.(25) have to respect the constraints given by the SIM of Eq.(22).

To summarize, through this section we mathematically described the physical problem, the impacts were modeled and the equations of motion obtained. The multiple scale method was applied to simplify and analytically solve the equations of motion. Under the assumption of 1:1 resonance between the LO and the VI-NES we found an expression for the Slow Invariant Manifold of the problem which provides some important information about the existence and the stability of all the possible solutions. Subsequently, by developing the equations of motion up to $\epsilon^{1}$-order, the fixed points were calculated, i.e. the steady-state solutions for a given external forcing. These points should respect the conditions imposed by the SIM to be actual physical solutions.

\section{Experimental and analytical results compari- son}

The purpose of this section is to find an analytical description and explanation to the VI-NES' behavior we observed experimentally. Particular attention is paid to the type of response the VI-NES may exhibit depending on the magnitude 
and frequency of the external forcing. Three levels of forcing amplitude are considered: low, medium and high. For each level the evolution of the system on the SIM as the external forcing frequency varies is analytically analyzed and compared to the experimental observations. For the analytic calculations, a coefficient of restitution $r=0.65$ is used. This is a typical value for an impact involving two metal bodies. Each case is presented as a graph on the $A_{\Omega}-\alpha$ plan where the curves (22) and (25) have been plotted. It is important to notice that eq.(25) can have real or imaginary solutions. In the graphs that follow only the real solutions are plotted as they represent the only physical fixed points.

\subsection{Low level forcing}

For a low level of forcing $\left(\ddot{x}_{e}=0.1 \mathrm{~g}\right)$ the activation threshold condition is not respected at any frequency and the VI-NES is never active. Experimentally the LO is not affected at all by the presence of the VI-NES. Analytically, as shown in fig.10, the fixed points evaluated by solving Eq.(25) (green curve) do not intersect the SIM curve and as a consequence no steady solution can be reached.

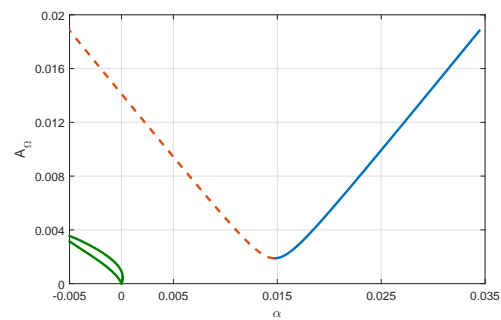

(a) $\Omega=0.970$

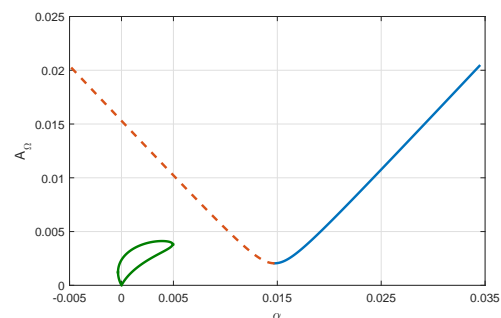

(c) $\Omega=1.010$

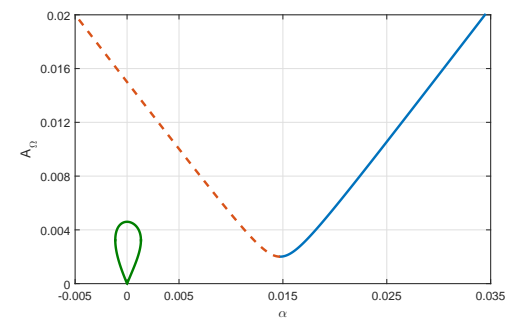

(b) $\Omega=1.000$

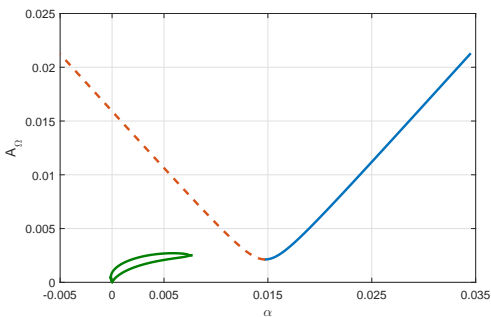

(d) $\Omega=1.030$

Figure 10: Evolution of the fixed points for a low level of external forcing $\ddot{x}_{e}=$ $0.1 \mathrm{~g}$ and four values of $\Omega$. The stable and unstable branches of the SIM are the blue and red-dashed lines respectively. The green curves are the solutions of (25). No intersections are present at any frequencies: the VI-NES is never active. 


\subsection{Medium level forcing}

Fig.11 shows the experimental spectrum of the primary mass displacement for a base acceleration $\ddot{x}_{e}=0.2 g$. The VI-NES stays idle up until the primary system's oscillations reach the activation threshold, it is active for a range of frequencies nearby the natural frequency of the LO and it turns idle again for higher frequencies of the forcing. However, the only type of response observed is the SMR, suggesting the VI-NES is not able to establish a stable-state solution. This assumption is actually confirmed by looking at the evolution of the fixed points on the SIM in Fig.12. Four different values of forcing frequency $\Omega=0.97 ; 1.00 ; 1.01 ; 1.03$ are shown. For $\Omega=0.97$ and $\Omega=1.03$ the isles (green curves) representing the fixed points do not intersect the SIM and the VI-NES is idle. For both $\Omega=1.00$ and $\Omega=1.03$ the fixed points curves have two intersection with the SIM. Nevertheless the points intersected are located on the unstable branch of the SIM: the solutions are then unstable. This is in agreement with the experimental observations which featured a Strongly Modulated Response. Analyzing the SMR, we can assume that the phase when the VI-NES is active is an unstable solution and therefore cannot be held. Subsequently the oscillations amplitude decreases until the VI-NES is not active anymore since the activation threshold condition is no more satisfied. Because of the constant external forcing the primary mass' oscillations get higher again and go beyond the activation threshold and the cycle starts over. Looking at Fig.4, It should be noticed that the cycles are each time different and appear to be chaotic. Recently Gendelman et al. [23] referred to this phenomenon as Chaotic Strongly Modulated Response (CSMR). A study of the chaotic SMRs is planned to be done in future work, where different chaos criteria might be applied to the experimental time series featuring SMR.

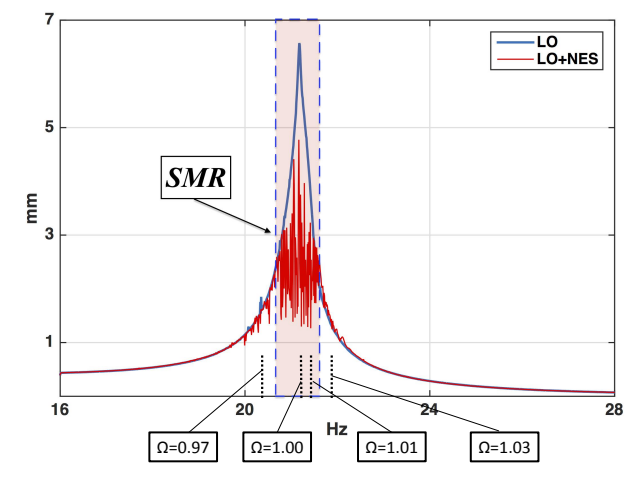

Figure 11: Experimental spectra of the LO's displacement with (red) and without (blue) the VI-NES. The regime of strongly modulated response (SMR) has been highlighted. 


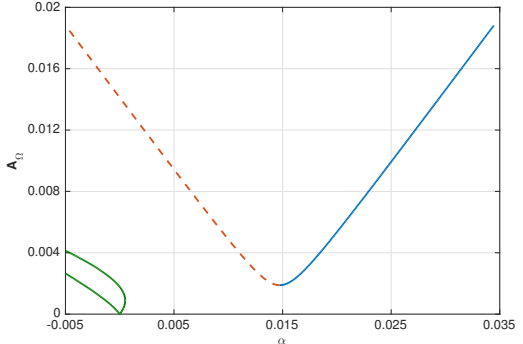

(a) $\Omega=0.970$ : Idle

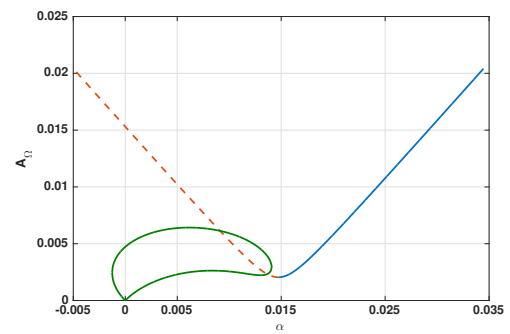

(c) $\Omega=1.010: \mathrm{SMR}$

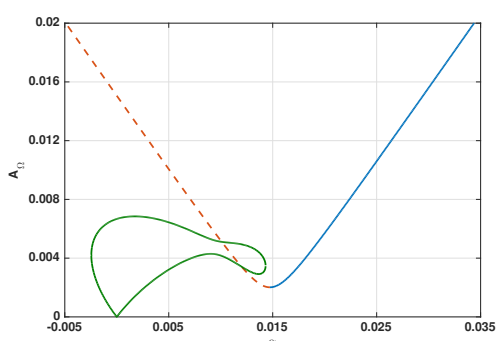

(b) $\Omega=1.002:$ SMR

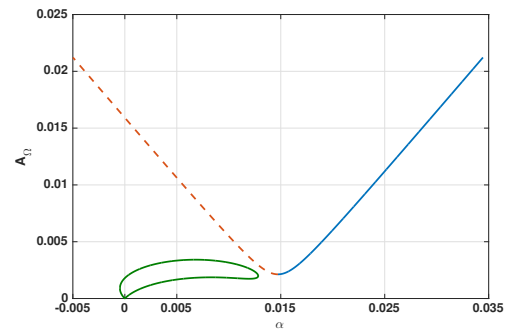

(d) $\Omega=1.030$ : Idle

Figure 12: Evolution of the fixed points for a medium level of external forcing $\ddot{x}_{e}=0.2 \mathrm{~g}$ and four values of $\Omega$. The stable and unstable branches of the SIM are the solid-blue and red-dashed lines respectively. The green curves are the solutions of (25). A SMR regime is the only type of solution that can be reached. This is in agreement with the experimental results of Fig.11. 


\subsection{High level forcing}

When the external base acceleration is $\ddot{x}_{e}=0.4 \mathrm{~g}$ the measured spectrum showed in Fig.13 presents two distinct zones featuring a CAR and a SMR. The VI-NES exhibits a CAR for most of the frequency range of activation and a SMR for a little portion just before turning back idle. The evolution of the fixed points on the SIM is illustrated in fig.14. At $\Omega=0.94$ we can see that a second fixedpoints isle appears but that still there are no points of intersection with the SIM. At $\Omega=0.97$ this second isle intersects twice the SIM on the stable and on the unstable branch. It means that at least one stable solution exists and a CAR can be established. When the frequency goes up, the stable point tends to lose its stability and eventually becomes unstable $(\Omega=1.04)$ as the isle of fixed points gets smaller. At $\Omega=1.06$ there are not intersections anymore and the VI-NES is idle again.

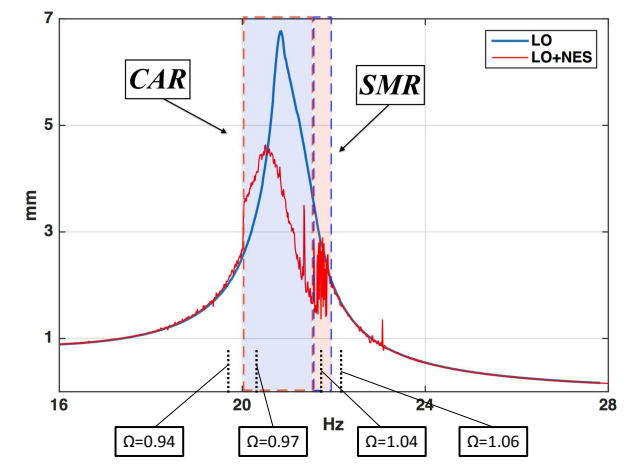

Figure 13: Experimental spectra of the LO's displacement with (red) and without (blue) VI-NES for $\ddot{x}_{e}=0.4 g$. The regimes of constant amplitude (CAR) and strongly modulated response (SMR) have been highlighted.

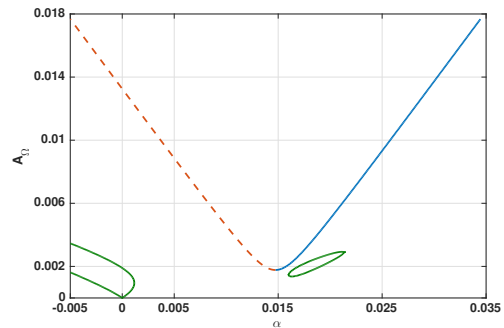

(a) $\Omega=0.940$ : Idle

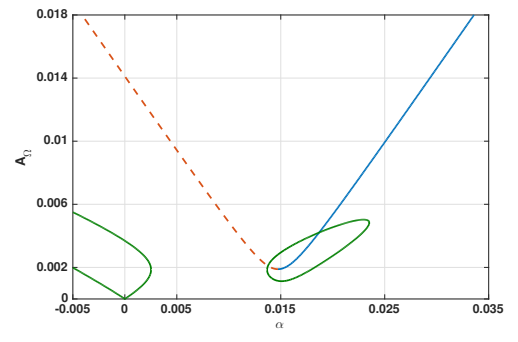

(b) $\Omega=0.970$ : CAR 


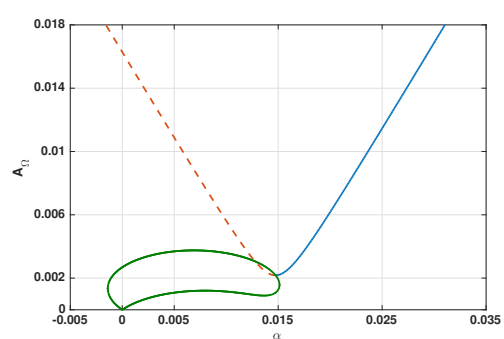

(c) $\Omega=1.040: \mathrm{SMR}$

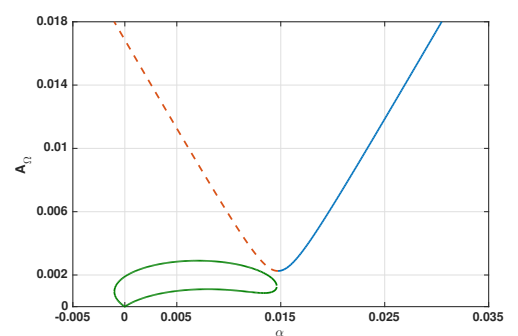

(d) $\Omega=1.060$ : Idle

Figure 14: Evolution of the fixed points for a high level of external forcing $\ddot{x}_{e}=0.4 \mathrm{~g}$ and four values of $\Omega$. The stable and unstable branches of the SIM are the solid-blue and red-dashed lines respectively. The green curves are the solutions of $(25)$. The system can have either stable $(\Omega=0.97)$ or unstable $(\Omega=1.04)$ fixed point intersections. CAR and SMR can both be observed.

\section{Influence of the tube's length and the mass ratio on the VI-NES response}

In this section the influence of the mass ratio $\varepsilon=m / M$ and the tube's length $L$ on the response of the VI-NES is studied. The case of low level forcing presented in the previous section is used to show how a variation in the parameters $m$ and $L$ can lead to a change in the VI-NES's response. This is done by means of the analytical model and by calculating the SIM and the fixed points of the problem.

In Sec.4.1 we saw how for a low level of forcing $\left(\ddot{x}_{e}=0.1 \mathrm{~g}\right)$ the activation threshold condition is not respected at any frequency and the VI-NES is never active. A way to have solutions for this level of forcing would be reducing the tube's length. If this happens the SIM would move left on the $A_{\Omega}-\alpha$ plan and the fixed points could intersect it. This example is illustrated in Fig.15 where the tube's length has been divided by two $\left(L=L_{0} / 2=7.5 \mathrm{~mm}\right)$.

The VI-NES is now capable to reach a Strongly Modulated Response state. If we go further in this analysis and keep reducing the length, there will be a point when the system gets to a Constant Amplitude Response. This example is shown in Fig. 16 where the length has been divided by three $\left(L=L_{0} / 3=5 \mathrm{~mm}\right)$

We can assume that the smaller the tube's length is the easier it is to reach a stable solution (CAR), i.e. even with a low level of external excitation. However, it is highly important to point out that this is a discussion about the qualitative responses the VI-NES may exhibit and not about the efficiency of the VI-NES as an absorber. Here the problem is studied from a kinematic point of view, not involving energy aspects. It means that reducing the length and reaching a stable fixed point may potentially make the VI-NES less efficient as an absorber. This point can be better understood by studying the effect of a mass ratio variation. 


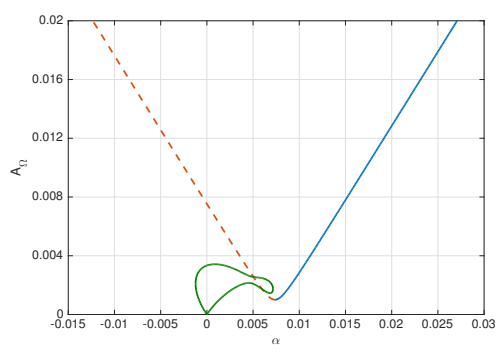

(a) $\Omega=1.002$

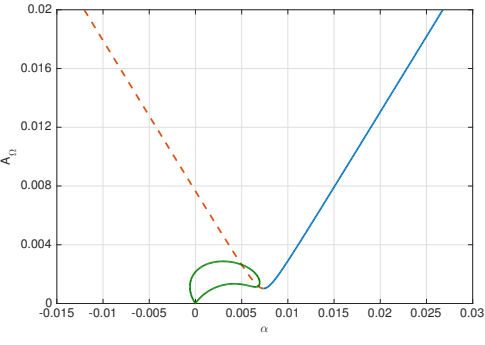

(b) $\Omega=1.010$

Figure 15: Same case as in Fig.10 of low level forcing but for a tube's length divided by two $\left(L=L_{0} / 2=7.5 \mathrm{~mm}\right)$. It can be seen that now a SMR regime is possible.

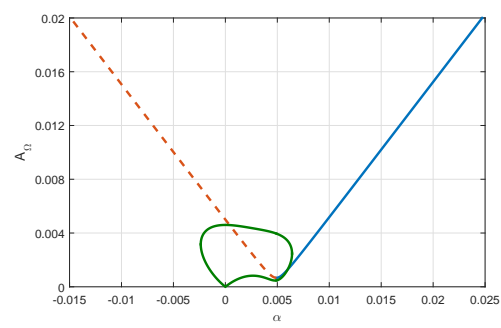

(a) $\Omega=1.002$

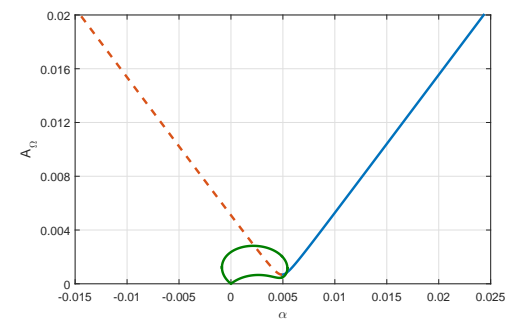

(b) $\Omega=1.010$

Figure 16: Same case as in Fig.10 of low level forcing but for a tube's length divided by three $\left(L=L_{0} / 3=5 \mathrm{~mm}\right)$. It can be seen that now a CAR regime is possible.

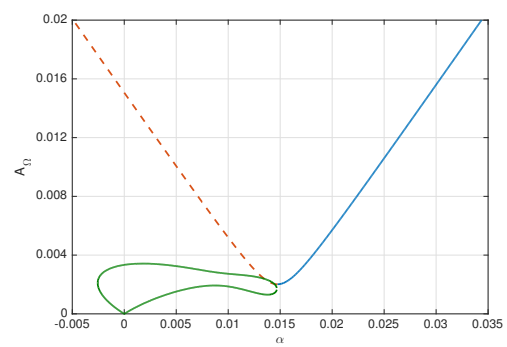

(a) $\varepsilon=0.20 \%-\Omega=1.002$

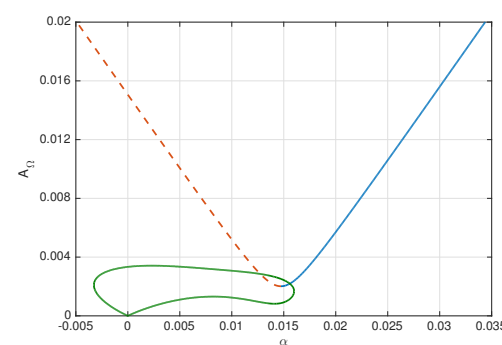

(b) $\varepsilon=0.15 \%-\Omega=1.002$

Figure 17: Same case as in Fig.10 of low level forcing but for a smaller mass ratio. It can be seen that a SMR and a CAR regime are possible.

A similar analysis is conducted in the case where the mass ratio $\varepsilon=m / M$ varies and the tube's length is kept constant. If the VI-NES mass is decreased 
to $\varepsilon=0.2 \%$, a Strongly Modulated Response can be established, as shown in Fig.17a. When the mass ratio is further decreased $(\varepsilon=0.15 \%)$ a Constant Amplitude Response state appears (see Fig.17b). It should be clear that the effect of reducing the mass ratio allows the VI-NES to reach a stable state but at the same time reduces the level of energy dissipated by the shocks and consequently the VI-NES's efficiency as an absorber. For this reason and because of the complex dependency of the problem on these parameters, an extensive energetic study will be carried out in future investigations.

\section{Conclusion}

In this work the dynamical behavior of a harmonically forced 1-dof LO coupled to a VI-NES has been explored. An experimental study has been carried out, in which the different regimes of response that may arise (constant amplitude and strongly modulated) and their dependence on the external forcing have been analyzed. The experimental results have also shown that the VI-NES is able to significantly reduce the resonance peak of the primary system even in the case of a considerably small mass ratio $(\epsilon=0.84 \%)$ and with no need of an optimized designing process. The Targeted Energy Transfer mechanism is observed.

Subsequently, the system has been analytically studied by means of the multiple-scale analysis. The Slow Invariant Manifold and the fixed points of the mathematical problem have been calculated. It has been shown that a threshold in the LO's amplitude for the TET to occur exist and how the SIM and the fixed points evolve as the external forcing term varies. The analytical model has allowed for a prediction of the response regime of the system and a good correlation with the experimental observations has been obtained.

These results complete the study begun in [24] by providing an exhaustive experimental and analytical analysis of the VI-NES and its dynamics when applied to a 1-dof linear oscillator. This work will constitute the basis for further investigations on the VI-NES: an energetic study and an optimization of the performances as a vibration absorber will be the next steps to reach a complete characterization of this kind of device.

\section{References}

[1] O. Gendelman, "Transition of energy to a nonlinear localized mode in a highly asymmetric system of two oscillators," Nonlinear Dynamics, vol. 25, pp. 237-253, 2001.

[2] A. F. Vakakis, O. V. Gendelman, L. I. Manevitch, and R. McCloskey, "Energy pumping in nonlinear mechanical oscillators part ii resonance capture," Journal of Applied Mechanics Transactions of the ASME, vol. 68, pp. $42-48,2001$. 
[3] A. Vakakis, O. Gendelman, L. Manevitch, and R. McCloskey, "Energy pumping in nonlinear mechanical oscillators i: dynamics of the underlying hamiltonian system," Journal of Applied MechanicsTransactions of the ASME, vol. 68, pp. 34-41, 2001.

[4] L. Manevitch, E. Gourdon, and C. Lamarque, "Parameters optimization for energy pumping in strongly nonhomogeneous 2 dof system," Chaos, Solitons and Fractals, vol. 31(4), pp. 900-911, 2007.

[5] G. Kerschen, Y. S. Lee, A. F. Vakakis, D. M. McFarland, and L. A. Bergman, "Irreversible passive energy transfer in coupled oscillators with essential nonlinearity," SIAM Journal on Applied Mathematics, vol. 66(2), pp. 648-679, 2005.

[6] D. McFarland, L. Bergman, and A. Vakakis, "Experimental study of nonlinear energy pumping occurring at a single fast frequencyexperimental study of non-linear energy pumping occurring at a single fast frequency," International Journal of Non-Linear Mechanics, vol. 40(6), pp. 891-899, 2005.

[7] E. Gourdon, N. Alexander, C. Taylor, C. Lamarque, and S. Pernot, "Nonlinear energy pumping under transient forcing with strongly nonlinear coupling: Theoretical and experimental results," Journal of Sound and Vibration, vol. 300(3), pp. 522-551, 2007.

[8] G. Kerschen, J. Kowtko, D. McFarland, L. Bergman, and A. Vakakis, "Theoretical and experimental study of multimodal targeted energy transfer in a system of coupled oscillators," Nonlinear Dynamics, vol. 47(1), pp. 285309, 2007.

[9] Y. Starosvetsky and O. Gendelman, "Strongly modulated response in forced 2dof oscillatory system with essential mass and potential asymmetry," Physica D, vol. 237(13), pp. 1719-1733, 2008.

[10] E. Gourc, G. Michon, S. Seguy, and A. Berlioz, "Experimental investigation and design optimization of targeted energy transfer under periodic forcing," Journal of Vibration and Acoustics, vol. 136, p. 021021, 2014.

[11] O. Gendelman and T. Bar, "Bifurcations of self-excitation regimes in a van der pol oscillator with a nonlinear energy sink," Physica D, vol. 239(3), pp. 220-229, 2010.

[12] Y. S. Lee, A. F. Vakakis, L. Bergman, D. McFarland, and G. Kerschen, "Suppressing aeroelastic instability using broadband passive targeted energy transfers, part 1: Theory," AIAA Journal, vol. 45(3), pp. 693-711, 2007.

[13] Y. S. Lee, G. Kerschen, D. McFarland, W. J. Hill, C. Nichkawde, T. Strganac, L. Bergman, and A. F. Vakakis, "Suppressing aeroelastic instability 
using broadband passive targeted energy transfers, part 2: Experiments," AIAA Journal, vol. 45(10), pp. 2391-2400, 2007.

[14] O. Gendelman, A. Vakakis, L. Bergman, and D. McFarland, "Asymptotic analysis of passive nonlinear suppression of aeroelastic instabilities of a rigid wing in subsonic flow," SIAM Journal on Applied Mathematics ., vol. 70(5), pp. 1655-1677, 2010.

[15] B. Vaurigaud, L. Manevitch, and C. Lamarque, "Passive control of aeroelastic instability in a long span bridge model prone to coupled flutter using targeted energy transfer," Journal of Sound and Vibration, vol. 330(11), pp. 2580-2595, 2011.

[16] O. Gendelman, "Targeted energy transfer in systems with non-polynomial nonlinearity," Journal of Sound and Vibration, vol. 315, pp. 732-745, 2008.

[17] O. Gendelman and C. Lamarque, "Dynamics of linear oscillator coupled to strongly nonlinear attachment with multiple states of equilibrium," Chaos, Solitons and Fractals, vol. 24, pp. 501-509, 2005.

[18] F. Georgiadis, A. F. Vakakis, D. M. McFarland, and L. A. Bergman, "Shock isolation through passive energy pumping caused by non-smooth nonlinearities," International Journal of Bifurcations and Chaos, vol. 15, pp. 1-13, 2005.

[19] I. Karayannis, A. F. Vakakis, and F. Georgiadis, "Vibro-impact attachments as shock absorbers," Proceedings IMechE, Journal of Mechanical Engineering Science, vol. 222, no. 222, pp. 1899-1908, 2008.

[20] Y. S. Lee, F. Nucera, A. F. Vakakis, D. M. McFarland, and L. A. Bergman, "Periodic orbits, damped transitions and targeted energy transfers in oscillators with vibro-impact attachments," Physica D, vol. 238, pp. 1868-1896, 2009 .

[21] F. Nucera, F. Lo Iacono, D. M. McFarland, L. A. Bergman, and A. F. Vakakis, "Application of broadband nonlinear targeted energy transfers for seismic mitigation of a shear frame: Part ii. experimental results," Journal of Sound and Vibration, vol. 313, pp. 57-76, 2008.

[22] O. Gendelman, "Analytic treatment of a system with a vibro-impact nonlinear energy sink," Journal of Sound and Vibration, vol. 331, pp. 4599-4608, 2012 .

[23] O. V. Gendelman and A. Alloni, "Dynamics of forced system with vibroimpact energy sink," Journal of Sound and Vibration, vol. 358, pp. 301-314, 2015 .

[24] E. Gourc, G. Michon, S. Seguy, and A. Berlioz, "Theoretical and experimental study of an harmonically forced vibro-impact nonlinear energy sink," Journal of Vibration and Acoustic, vol. 137, p. 031008, 2014. 
[25] A. H. Nayfeh, Perturbation methods. WileyVCH Verlag, 2004.

[26] G. X. Li, R. H. Rand, and F. C. Moon, "Bifurcations and chaos in a forced zero-stiffness impact oscillator," Journal of Nonlinear Mechanics, vol. 25(4), pp. 417-432, 1990. 